\title{
Wie aus digitalen Services Wert entsteht: Interaktionen richtig gestalten
}

\author{
Manuel Geiger $\cdot$ Susanne Robra-Bissantz $\cdot$ Michael Meyer
}

Eingegangen: 10. Dezember 2019 / Angenommen: 14. März 2020 / Online publiziert: 24. März 2020

(C) Der/die Autor(en) 2020

Zusammenfassung In der digitalen Wirtschaft finden sekündlich mannigfaltige digitale Interaktionen zwischen Anbietern und ihren Kunden statt. Sie entscheiden über die Auswahl von Produkten und Dienstleistungen und werden daher für Unternehmen zu einem wesentlichen Erfolgsfaktor. Trotzdem fehlt es bislang in Theorie und Praxis an Modellen und Ansätzen, die diese Interaktionen beschreiben, erklären und damit als Grundlage ihrer Gestaltung dienen können. Dieser Beitrag sieht Interaktionen des Anbieters mit seinen Kunden als digitale Services, die den Kunden unterstützen: bei der Suche nach Produkten und Dienstleistungen sowie der Auswahl und Nutzung derer. Dazu wird zunächst der Wert der digitalen Interaktion erarbeitet, bezeichnet als Value in Interaction. Dieser entsteht auf einer Beziehungsebene, der Matching-Ebene und der Ebene der eigentlichen Dienstleistung. Auf jeder dieser Ebenen wird ein Wert geschaffen - der Beziehungswert, der Matching-Wert und der Dienstleistungswert. Mit konkreten Handlungsempfehlungen wird die Praxis bei der aktiven Gestaltung von Interaktionen in Form von digitalen Interaktionsservices unterstützt. Damit ist sichergestellt, dass nicht nur das Angebot, sondern auch die einzelnen Interaktionen in ihrer Gesamtheit auf den einzelnen Kunden und seine Bedürfnisse angepasst werden.

Schlüsselwörter Digitale Transformation · Value in Interaction · Service Design · Service Interaktion · Digitale Services

M. Geiger $(\bowtie) \cdot$ S. Robra-Bissantz $\cdot$ M. Meyer

Abteilung Informationsmanagement, Institut für Wirtschaftsinformatik, Technische Universität Braunschweig, Mühlenpfordtstr. 23, 38106 Braunschweig, Deutschland

E-Mail: m.geiger@tu-bs.de 


\section{How to Create Value from Digital Services: Designing Interactions Properly}

Abstract In the digital economy, there are multiple digital interactions every second between providers and their customers. They decide which products and services to choose and therefore become a key success factor for companies. Nevertheless, up to now there is a lack of models and approaches in theory and practice that can describe and explain these interactions and thus serve as a basis for their design. This paper views interactions of the provider with its customers as digital services that support the customer: in the search for as well as the selection and use of products and services. For this purpose, the value of digital interaction is initially developed, referred to as Value in Interaction. This consists of the relationship layer, the matching layer and the service layer. On each of these layers, a value is created-the relationship value, the matching value and the service value. With concrete recommendations for action, the practice is supported in actively designing interactions in the form of digital interaction services. This is to ensure that not only the offer but also the individual interactions as a whole are adapted to the individual customer and his needs.

Keywords Digital Transformation - Value in Interaction - Service Design - Service Interaction · Digital Services

\section{Einleitung}

Der Mensch hat in der Gestaltung seines eigenen Lebens durch die digitale Transformation, gespeist aus technologischem Fortschritt und der Nutzung dieser Technologien, mehr Freiheit und Möglichkeiten erhalten. Er kann zu jeder Zeit und an jedem Ort online Produkte kaufen und Dienstleistungen in Anspruch nehmen. Darunter auch Produkte und Dienstleistungen zu denen er zuvor keinen Zugang hatte oder die es schlicht nicht gab (Heinemann 2013). Die hohe Anzahl an verfügbaren Angeboten und die damit verbundenen Informationen sind Vorteile, die das Internet erst ermöglicht hat (Stichwort: Longtail). Trotzdem muss das nicht zwangsläufig auch immer mit einem Mehrwert für den Kunden einhergehen. Auch wenn es eine Vielzahl digitaler Services in Form von Tools und Mechanismen gibt, die dem Kunden helfen, sich bei einer Vielzahl von Angeboten zurechtzufinden (z. B. Filter, Suchwerkzeuge) - zu viel Auswahl hat eine lähmende Wirkung (Schwartz 2004). Eine sogenannte „Choice Overload“ tritt ein, wenn die Anzahl der Möglichkeiten (hier: Informationen) die kognitiven Fähigkeiten des Kunden übersteigt, was insbesondere in der digitalen Welt der Fall ist (Wan et al. 2003). Die Folgen sind beispielsweise eine verminderte Motivation eines der verschiedenen Angebote zu wählen (Iyengar und Lepper 2000) und ein gestiegenes Risiko eine nicht optimale Entscheidung zu treffen. Der Kunde ist von der Vielzahl an Informationen und der damit einhergehenden Informationsverarbeitung überfordert (Wan et al. 2003).

Schuld an der Informationsfülle sind aufgrund des Technologiefortschrittes im Rahmen der Digitalisierung die vielen neuen Interaktionsmöglichkeiten und das da- 
mit gesteigerte Angebot an digitalen Services. Über digitale Schnittstellen greifen Kunden auf das digitale Angebot der Unternehmen zu, sie nutzen digitale Vermittlungsplattformen oder Vergleichsangebote, informieren sich in Webshops oder werden über Apps wie TripAdvisor, AirBnB etc. angesprochen. Schließlich entscheidet dann die mehr oder weniger gelungene digitale Interaktion darüber, welche Produkte oder Dienstleistungen der private Kunde wählt. Trotz dieser entscheidenden Rolle in der Geschäftsbeziehung liegt der Fokus in der Praxis im Wesentlichen in der Gestaltung neuer und besserer USPs eines Produktes oder einer Dienstleistung. Wenn es um Kommunikation geht wird es der Werbung oder dem Marketing zugeordnet. Die aktive Gestaltung der (IT-gestützten) Interaktion mit dem Kunden erfährt hingegen wenig Aufmerksamkeit. Jedoch reicht es nicht mehr aus, wenn ein Restaurant „nur“ sehr gut kochen und bedienen kann. Es reicht nicht mehr aus, wenn ein Autobauer "nur" sehr gute Autos baut. Interaktion ist der Klebstoff, der Akteure miteinander verbindet (Fyrberg und Jüriado 2009) und damit essentiell, um mit dem eigenen Angebot für den Kunden einen Wert schaffen zu können. Doch was zeichnet eine gute Interaktion aus?

Im Marketing wurden mit dem Relationship Marketing, der Service Logic (SL) oder auch der Service Dominant Logic (S-D logic) Theorien entwickelt, die dem Unternehmen in einer sehr stark kundenzentrierten Sichtweise aufzeigen, wie es Produkte und Dienstleistungen erfolgreich gestalten kann. Diese servicezentrierten Theorien (im Weiteren als Service Logik bezeichnet) haben die Denkweise darüber verändert, was im wirtschaftlichen Handeln passiert. Im Zentrum steht dabei der Wert des Angebots für den Kunden, der in dieser Logik immer durch eine Dienstleistung ${ }^{1}$ entsteht. Es ist dann nicht mehr der Anbieter mit seinem Produkt, der einen Wert schafft, sondern der Wert entsteht daraus, dass sich der Kunde die Kompetenzen des Anbieters ${ }^{2}$ nutzbar macht (Value in Use). Dieser Wert bemisst sich alleine aus der Wertschöpfung, die der Kunde daraus zieht. Die dedizierte Berücksichtigung des Value in Use einer Dienstleistung hat sich als Ansatzpunkt für erfolgreiche Marktangebote bewährt. Es liegt daher für die Wirtschaftsinformatik nahe, auch digitale Interaktionen an dem Wert zu bemessen, die sie für den Kunden bieten. Im Rahmen dieses Beitrages wird daher, basierend auf der bestehenden theoretischen Basis der Service Logik, ein Value in Interaction eingeführt, der durch digitale Services in der Interaktion entstehen kann und auf besonderen Kompetenzen des Anbieters in der Interaktion beruhen. Aufbauend auf dem potenziellen Wert einer Interaktion ergeben sich Ansatzpunkte zur Gestaltung dieser digitalen Services. Damit unterstützt die neue Sichtweise alle Akteure auf digitalen Märkten, indem sie den Kunden mit seinen Such-, Entscheidungs- und Auswahlaktivitäten als Menschen sieht und seine dementsprechenden Bedürfnisse in den Mittelpunkt der Entwicklung neuer digitaler Services stellt.

Nachdem im zweiten Abschnitt das Value in Interaction Modell eingeführt und erläutert wird, wird in Abschn. 3 kurz die Bedeutung des Modells aus Sicht eines Unternehmens beleuchtet. In Abschn. 4 werden erste Handlungsempfehlungen für die Praxis aufgestellt, bevor dann abschließend in Abschn. 5 ein Fazit gezogen wird.

\footnotetext{
1 Auch Produkte sind in dieser Logik den (indirekten) Dienstleistungen zuzuordnen.

2 Gemäß der Service Logik sind hiermit sowohl Kompetenzen als auch Ressourcen gemeint.
} 


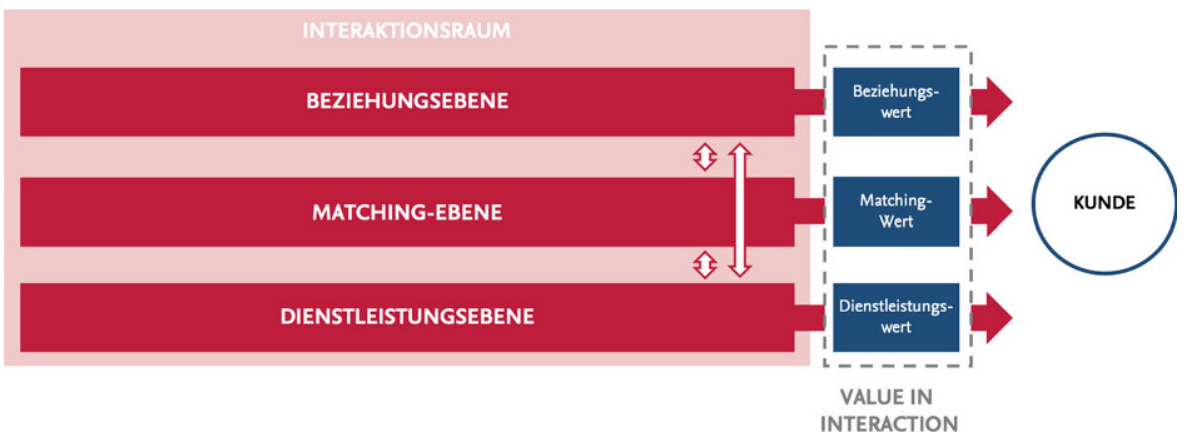

Abb. 1 Value in Interaction Modell. (Quelle: eigene Darstellung)

\section{Der Value in Interaction und seine Bestandteile}

Grundlage jeglicher Interaktion ist eine Verbindung zwischen den Akteuren in einem gemeinsamen Interaktionsraum, der von einem Anbieter zur Verfügung gestellt und von einem Kunden betreten werden kann. Ein solcher Interaktionsraum kann das physische Geschäft eines Einzelhändlers sein, aber auch ein digitaler Raum, wie beispielsweise eine Website, eine App (z.B. Nike+). Oder auch ein über digitale Medien auf Seiten des Anbieters automatisierter Informationsfluss zwischen den Akteuren, wenn beispielsweise der Erinnerungsservice des Arztes auf eine anstehende Vorsorgeuntersuchung aufmerksam macht. Durch Interaktionen in diesem Interaktionsraum haben die Akteure die Möglichkeit, sich mit dem jeweils anderen Akteur auseinanderzusetzen und diesen beziehungsweise sein Verhalten zu beeinflussen. Das gelingt jedoch nur dann, wenn die Interaktion auch vom Kunden als wertvoll angesehen wird. Ziel der Anbieter ist es, Interaktionsräume mit Kunden zu öffnen, möglichst auszuweiten, beziehungsweise immer wieder eröffnen zu können. Nur so können diese mit wertvollen Interaktionen in Form von digitalen Services gefüllt werden.

Der Value in Interaction entsteht innerhalb eines solchen Interaktionsraumes. Er entwickelt sich durch und während der Interaktion, er entfaltet seine Wirkung im Moment und beeinflusst dadurch die weiteren Prozesse der gemeinsamen Wertschöpfung (Co-Creation). Analysiert und gruppiert man Potenziale dafür, was für einen Kunden in einer Interaktion wertvoll sein kann, so ergeben sich Werte auf drei unterschiedlichen Ebenen: der Beziehungs-, der Matching- und der Dienstleistungsebene (s. Abb. 1). Der offensichtliche Wert, den ein Kunde aus digitalen Interaktionen mit dem Anbieter ziehen kann, ist die für ihn am besten geeignete Dienstleistung zu finden und möglichst bequem zu erwerben. Dieser Wert wird als Matching-Wert bezeichnet. Dafür müssen die beim Anbieter vorhandenen Kompetenzen bestmöglich mit den Bedürfnissen des Kunden in Einklang gebracht werden. Hat der Kunde sich für eine Dienstleistung, beispielsweise für die Buchung einer Reise, entschieden, dann können weitere digitale Services vor, während oder nach der Reise dazu führen dass die Dienstleistung möglichst gut gelingt: der Kunde wird rechtzeitig daran erinnert die notwendigen Impfungen zu erledigen oder er erhält das Angebot seine Erinnerungen in einem Fotobuch zu verewigen. Alle damit zusammenhängen- 
den Interaktionen wirken auf die Dienstleistung an sich und damit auf den Value in Use (Service Logik) seiner Reise. Der entsprechende Wert wird hier als Dienstleistungswert bezeichnet. Während allen Interaktionen verändert sich die Beziehung zwischen Kunde und Anbieter. Fühlt der Kunde sich beispielsweise gut verstanden, hat er das Gefühl, dass der Anbieter auf seine Bedürfnisse eingeht und steigt damit sein Vertrauen in den Anbieter, dann entsteht ein so genannter Beziehungswert.

Alleine das Vorhandensein einer Interaktion allerdings führt nicht zu Wert. Vielmehr kommt es auf die Qualität der Interaktion an (Fyrberg und Jüriado 2009). So wird eine von mittelmäßigen oder auch negativen Aspekten geprägte Interaktion (fehlende Qualität) negative Auswirkungen auf die Zufriedenheit des Kunden mit der Dienstleistung oder dem Produkt haben. Ein Unternehmen sollte seine Interaktionen somit immer bewusst als digitale Services gestalten. Um wertvolle Interaktionen gestalten zu können und nutzlose oder gar negativ wirkende Interaktionen zu vermeiden, müssen heutige Unternehmen daher Kompetenzen auf allen drei Ebenen vorweisen. Auf der Beziehungsebene handelt es sich dabei insbesondere um Kollaborations- und soziale Kompetenzen. Dienstleistungs-Kompetenzen sind die Fähigkeiten, genau zu wissen, welchen Wert ein potenzieller Kunde aus dem Angebot zu ziehen gedenkt. Derartige Kompetenzen liegen in den meisten Unternehmen vor - obwohl sie deutlich mehr vom Anbieter verlangen, als ein gutes Produkt zu entwickeln. Matching-Kompetenzen gehen darüber hinaus. Das Unternehmen muss Entscheidungsprozesse seiner Kunden kennen, es muss wissen, welche Situationen des Kunden zu welchen Dienstleistungsbestandteilen passen und diese zuordnen.

\subsection{Praxisbeispiele}

Die drei Ebenen des Value in Interaction und die daraus jeweils entstehenden Werte sind eng miteinander verknüpft und beeinflussen die jeweils anderen Ebenen. Im weiteren Verlauf sollen zwei Praxisbeispiele eine positive sowie eine negative Wertgenerierung auf den drei Ebenen veranschaulichen und im weiteren Verlauf zur Erläuterung des Modells dienen.

\subsubsection{Praxisbeispiel 1: Störungsmeldung bei einem Telekommunikationsunternehmen}

Situation: kompletter Kabelanschluss (Internet, Telefon, TV) ausgefallen Der Kunde bemerkt die wiederholte Störung seines Anschlusses und versucht, sich über die Webseite (offener Interaktionsraum) des Anbieters über das weitere Vorgehen zu informieren. Eine Störungsabfrage an der Adresse kommt zu dem Ergebnis, dass keine Störung vorliegt. Sofern dies dem Kunden nicht weiterhelfen sollte, eröffnet der Anbieter an dieser Stelle richtigerweise direkt einen weiteren Interaktionsraum: den Anruf bei einer Störungshotline. Dort leitet eine automatisierte Computerabfrage den Kunden durch mehrere Fragen. Ergebnis laut Anbieter ist auch hier, dass an diesem Anschluss keine Störung vorliegt. Das Gespräch wird daraufhin vom Anbieter automatisch beendet. Der Kunde wiederum versucht, sein Problem im Rahmen eines weiteren offenen Interaktionsraumes, einem Chat mit einem Chatbot, lösen zu lassen. Dieser reagiert allerdings nach kurzer Konversation nicht mehr (Abb. 2a). 


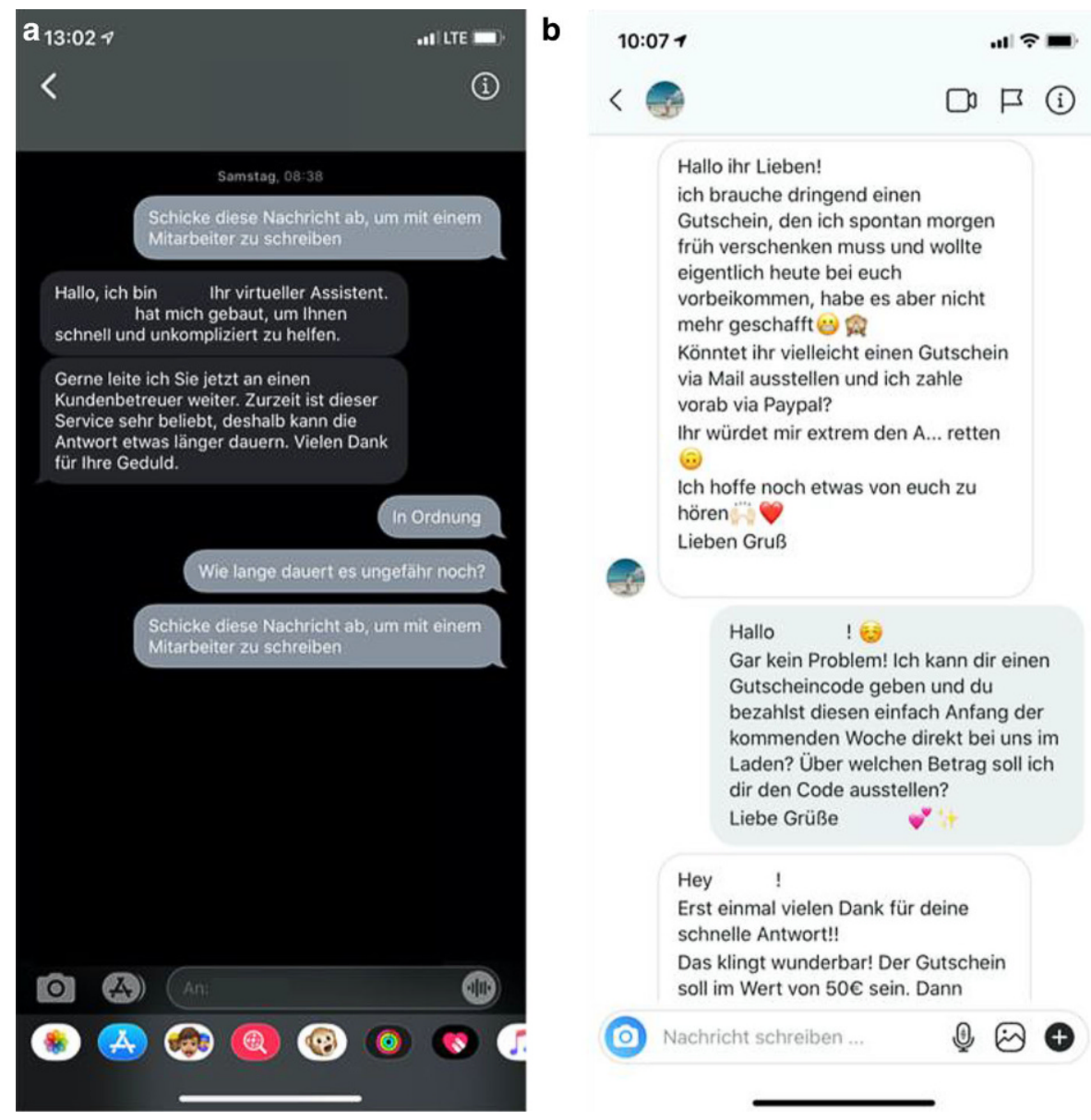

Abb. 2 Positive und negative Praxisbeispiele. (Quelle: eigene Aufnahmen)

Dieses Beispiel zeigt, dass alle Interaktionen des Anbieters nicht dazu geeignet waren, auf den verschiedenen Ebenen des Value in Interaction Modells einen positiven Wert zu erzeugen. Weder können die Interaktionen auf der Beziehungsebene positiv wirken (automatisierte Services), noch erfolgt eine adäquates Matching (z.B. Kommunikationsstil anpassen). Auf dieser Basis kann dementsprechend noch nicht einmal der originäre Wert einer Störungsbehebung (Dienstleistungsebene) durch den Anbieter erfolgen und somit auch kein positiver Value in Interaction entstehen.

\subsubsection{Praxisbeispiel 2: Geschenksuche am späten Samstagabend für Sonntag}

Situation: Kundin benötigt Samstagabend nach Ladenschluss noch ein Geschenk, um es sonntags verschenken zu können Eine Kundin wendet sich über Instagram (offener Interaktionsraum) nach Ladenschluss mit ihrem Anliegen an die Händlerin. Diese nutzt den offenen Interaktionsraum und ermöglicht der Kundin 
das Verschenken eines Gutscheins, in dem sie ihr direkt auf Vertrauensbasis einen digitalen Gutschein mit einem Gutscheincode über den gewünschten Betrag zukommen lässt. Die Kundin müsste dann nur zu Beginn der Woche in das Ladengeschäft kommen, um dort zu bezahlen. Die Kundin ist begeistert und nimmt das Angebot an. Auch wenn der Interaktionsraum des lokalen Geschäfts bereits geschlossen ist, stellt die Händlerin das Potenzial eines offenen Interaktionsraum bereit. Sie bringt der Kundin in dem persönlichen Chat Vertrauen (Beziehungsebene) entgegen, erkennt schnell, wie sie das Kundenproblem lösen kann (Matching-Ebene) und erbringt den Service direkt über einen für die Kundin situationsspezifisch passenden Kanal (Dienstleistungsebene). Mehr noch: sie entwickelt in der Interaktion eine Lösung für das Problem der Kundin mit einer passenden Dienstleistung, dem Gutschein. Dadurch entsteht auf allen drei Ebenen ein positiver Wert, was zu einem positiven Value in Interaction führt. Zudem eröffnet die Händlerin durch den folgenden Ladenbesuch direkt wieder einen Interaktionsraum.

\subsection{Beziehungswert}

Eine Beziehung zwischen Akteuren stellt per se bereits einen Wert für die Akteure dar (Ravald und Grönroos 1996), denn der Anbieter wurde vom Kunden kontaktiert, sodass er diesem eine Dienstleistung anbieten kann. Der Beziehungswert jedoch kann sowohl positiv als auch negativ entwickelt werden. Das Öffnen eines gemeinsamen Interaktionsraums als Voraussetzung für eine gemeinsame Wertschöpfung (Co-Creation) wird immer dann deutlich erleichtert, wenn zwischen den Akteuren bereits eine positive Wahrnehmung der Beziehung besteht. Bei den aktuellen Beispielen ist es also erstmal von Vorteil, wenn die bisherigen Interaktionen des Kunden mit dem Anbieter einen positiven Beziehungswert geschaffen haben. Ein vertrauensvolles Verhältnis zwischen Kundin und Händlerin (Praxisbeispiel 2) sowie die Verfügbarkeit eines offenen Interaktionsraumes (Instagram) ermöglichen die Interaktion. In der Interaktion kann der Beziehungswert ausgehend von allen drei Ebenen steigen oder auch sinken. Fühlt sich die Kundin auf der Beziehungsebene beispielsweise durch die zeitnahe Antwort sowie die Ansprache und den eingesetzten Kommunikationsstil mit Smileys ernst genommen und wertgeschätzt, hat das einen positiven Einfluss auf den wahrgenommenen Beziehungswert.

Auch Daten aus vorangegangenen Interaktionen können helfen, die Beziehungsebene zu stärken. Wenn in Praxisbeispiel 1 bekannt ist, dass es bei dem Kunden bereits vor kurzem eine längerfristige Störung gab, sollte der Kommunikationsstil angepasst werden. So kann das Verständnis für eine Situation auf beziehungsrelevante Art und Weise zum Ausdruck gebracht werden. Je länger eine Beziehung dauert, desto größer ist das Potenzial den Kunden und seine Bedürfnisse kennen zu lernen. Somit kann eine gelungene Interaktion durch Lerneffekte (Grönroos und Ojasalo 2004) eine Verbesserung für zukünftige Interaktionen bedeuten (Grönroos 2011) und die Matching-Ebene einen positiven Beitrag zur Entstehung des Beziehungswerts beitragen.

Wird nun, wie im Praxisbeispiel 2, das Anliegen der Kundin im Rahmen dieser Interaktion gelöst, so festigt die Händlerin potenziell ihre Rolle als Problemlöserin. Das hat wiederum einen positiven Einfluss auf die Beziehung. Durch die Erfüllung 
oder das Übertreffen der Erwartungen bei der eigentlichen Dienstleistung kann also auch die Dienstleistungsebene die Beziehungsebene positiv beeinflussen. Grundsätzlich spielt also auf der Beziehungsebene die soziale Verbundenheit, hervorgerufen durch das Antizipieren von Werten und Bedürfnissen, eine große Rolle.

\subsection{Matching-Wert}

Auf der Matching-Ebene findet sich der Wert der Interaktionen, der laut der Literatur die Dichte der Dienstleistung erhöhen kann (Normann 2001; Lusch und Nambisan 2015) und damit zu einem bestmöglichen Angebot für den Kunden führt. Sie stellt somit sicher, dass der Anbieter, wenn er über die passenden Kompetenzen für die spezifischen Bedürfnisse des Kunden verfügt, diese auch so einsetzen kann, dass die bestmögliche Dienstleistung für den Kunden daraus entsteht. Der Matching-Wert entsteht also dann, wenn die Fähigkeiten genutzt werden, um die richtigen digitalen Services in der Interaktion (z.B. Beratung) zielgerichtet einzusetzen, um die Bedürfnisse des Kunden herauszufinden. Das ist Voraussetzung dafür, dass ein Wert während des Dienstleistens (Dienstleistungswert) entsteht. Im Falle des ersten Praxisbeispiels wäre es zielführend gewesen, wenn Chatbot, Website oder Call-Center das eigentliche Anliegen des Kunden verstanden und adressiert hätten - z.B. eine wiederhergestellte Erreichbarkeit. So aber konnte durch ein fehlendes Matching kein adäquates Dienstleistungsangebot und somit auch kein Dienstleistungswert generiert werden. Im zweiten Praxisbeispiel konnte die Händlerin über ihren digitalen Kanal das Bedürfnis der Kundin gut verstehen. Das Matching ist gelungen. Vielmehr konnte die Händlerin durch das Angebot eines zusätzlichen Service-Bestandteils bezüglich der Bezahlung (,... [bezahl] [...] einfach Anfang der kommenden Woche direkt bei uns [...]") das Matching sogar auf Bestandteile ihrer Dienstleistung ausweiten, die sie bislang nicht im Programm hatte. Die Matching-Ebene und der daraus entstehende Wert ist somit von großer Bedeutung für den Dienstleistungswert. Damit kann aus dem Matching auch für die vorgelagerte Phase der Dienstleistungskonzeption ein Wert entstehen: nämlich dann, wenn das Matching auf Basis der verfügbaren Dienstleistungen nicht zu einem passenden Angebot führt. Der Anbieter kann dann seine zukünftigen Dienstleistungen besser an die Bedürfnisse der Kunden anpassen.

Je näher sich die beiden Akteure auf der Beziehungsebene stehen, desto größer ist die Möglichkeit das Dienstleistungsangebot so anzupassen, dass es den größtmöglichen Wert für den Kunden schafft. Hat die Kundin also einen positiven Beziehungswert mit dem Anbieter, was gleichbedeutend mit einer oder mehreren vorangegangenen, erfolgreichen Interaktion auf der Beziehungsebene ist, so ist er tendenziell offener die für das Matching wichtigen Informationen mit dem Anbieter zu teilen. Das bedeutet, dass Beziehungen, die über einen längeren Zeitraum aufgebaut wurden und bei denen bereits eine soziale Nähe entstanden ist, von der Verknüpfung der Interaktionen profitieren. Somit wirkt sich die Beziehungsebene direkt auf die Matching-Ebene aus. Gleichzeitig trägt eine auf der Dienstleistungsebene die Erwartungen erfüllende oder übertreffende Dienstleistung dazu bei, dass in folgenden Interaktionen das Matching noch genauer durchgeführt werden kann. 


\subsection{Dienstleistungswert}

Die Dienstleistungsebene beschreibt, wie die Interaktion die eigentliche Dienstleistung beeinflusst und im Dienstleistungswert resultiert. Dabei bilden die serviceorientierten Denkweisen des Marketings die Grundlage (Service Logik). Die Kompetenzen des Anbieters enthalten an sich noch keinen Wert, sondern stellen die Value Proposition des Anbieters (z.B. Aufmerksamkeit, Wissen, Reputation) dar (Grönroos und Voima 2013). Sobald der Kunde diese nutzt, um für sich seinen eigenen Wert zu kreieren, nimmt der Anbieter an der Wertschöpfung des Kunden teil - in einem Interaktionsprozess in Co-Creation (Grönroos und Ravald 2009; Grönroos und Voima 2013).

Die Händlerin aus dem positiven Praxisbeispiel hat es mit ihren Matching-Kompetenzen geschafft, zusammen mit der Kundin in Co-Creation in einer geeigneten Interaktion das richtige Angebot (hohe Dichte) zu kreieren. Damit sind die Voraussetzungen einer Wertschöpfung auf der Dienstleistungsebene gegeben. Das führt wiederum durch ein gesteigertes Vertrauen in die Kompetenzen der Händlerin zu einem positiven Einfluss auf die Beziehungsebene. Bemerkt der Kunde wie in dem negativen Praxisbeispiel hingegen, dass die Kompetenzen auf der Matching-Ebene nicht ausreichen, um das bestmöglichste Ergebnis zu erreichen, so hat das eine negative Auswirkung auf den Dienstleistungswert. Die indirekte Wirkung über die Beziehungsebene wirkt sich in diesem Fall sogar noch als Verstärker aus. Da es der Telekommunikationsanbieters nicht schafft die Kommunikation auf die persönlichen Bedürfnisse des Kunden abzustimmen (s. Abschn. 2.2), leidet der Beziehungswert, was wiederum einen zusätzlichen negativen Einfluss auf die Dienstleistungsebene hat. Demgegenüber ist davon auszugehen, dass eine gelungene, beziehungsorientierte Interaktion den Beziehungswert erhöht. Dass sich die Kundin aus dem zweiten Praxisbeispiel auf der Beziehungsebene verstanden und wertgeschätzt fühlt hat demzufolge einen positiven Einfluss auf die Dienstleistungsebene.

\section{Bedeutung des Value in Interaction für Unternehmen}

Die serviceorientierten Theorien des Marketings sehen das Dienstleisten als gegenseitigen Prozess des Austausches von Kompetenzen oder Ressourcen. Damit verschmelzen die Rollen von Kunde und Anbieter zu Akteuren, die jeweils in ihrem Kontext die am besten zu ihrer Wertschaffung passenden Kompetenzen wählen (Vargo und Lusch 2008). Auch der Kunde bringt also einen Wert für den Anbieter in die Dienstleistung mit ein. Dies ist in den bisherigen Beispielen auch bereits ersichtlich, erhält die Händlerin doch beispielsweise Kundendaten, die sie zukünftig wiederum einsetzen kann. Dementsprechend gelten die bis hierhin diskutierte Werte (Beziehungswert, Matching-Wert und Dienstleistungswert) für den Anbieter gleichermaßen.

Geht man davon aus, dass Interaktionen immer dann stattfinden, wenn alle beteiligten Akteure ihren Dienstleistungswert erhöhen können, dann besteht der große Unterschied zwischen der Dienstleistungsebene und der Beziehungsebene darin, dass sich der Dienstleistungswert bei den Akteuren sinnvollerweise unterscheiden muss. 
Denn jeweils sollen die Kompetenzen eines Akteures zum Wert des anderen beitragen. Ein Akteur erfährt damit einen Wert genau darin, dass er andere Kompetenzen erhält, als er selber bereits besitzt. Der Beziehungswert hingegen kann und muss langfristig in vielen Bereichen gleich sein - er umfasst beispielweise das gegenseitige Vertrauen, ein gegenseitiges Commitment und die gegenseitige Zufriedenheit. Gewöhnlich ist damit davon auszugehen, dass der aus den Interaktionen resultierende Beziehungswert für beide Partner in gleicher Weise steigt oder sinkt. Fyrberg und Jüriado (2009) bezeichnen in diesem Zusammenhang die Balance von Vertrauen als entscheidenden Faktor im Prozess von Interaktionen zwischen gleichberechtigten Akteuren. Besteht die Gefahr, dass durch eine Asymmetrie bei der Einbringung und der Intensität eines Akteurs eine Disbalance bei der Wertegenerierung entsteht, sollte das Verhalten des anderen Akteurs gespiegelt werden, um einem geringen oder gar negativen Beziehungswert entgegenzuwirken.

\section{Handlungsempfehlungen}

Aus Sicht der Praxis stellt sich nun die Frage, wie das Value in Interaction Modell konkret umgesetzt werden kann. Der wesentliche Faktor ist hierbei, insbesondere die aktive Gestaltung der digitalen Interaktion mit ihren unterschiedlichen Ebenen. Um das zu ermöglichen, muss das Unternehmen seine Interaktionskompetenzen in den Co-Creation Prozess mit dem Kunden einbringen. Um diese Kompetenzen zu entwickeln und einzusetzen, bedarf es der Differenzierung in die unterschiedlichen Ebenen einer Interaktion (s. Abschn. 2). Grundsätzlich stellt sich in der operativen Anwendung des Value in Interaction Modells zuerst die Frage, ob man als Anbieter einen offenen Interaktionsraum bereitstellt. Dabei ist es von großer Bedeutung, ob dieser Interaktionsraum von dem Kunden auch als solcher gesehen wird. Ohne einen offenen Interaktionsraum kann keine Interaktion und somit auch kein Value in Interaction entstehen. Dabei muss der Anbieter versuchen, den Interaktionsraum so lange wie möglich offen zu halten oder ihn wieder zu öffnen. Je länger er den Kunden in seiner Wertschaffung interaktiv begleiten kann, den Interaktionsraum also geöffnet lässt, desto mehr Optionen bieten sich ihm, an dieser auch teilzunehmen und seine Kompetenzen einzubringen.

Grundsätzlich sollten Unternehmen bei dem Einsatz des Value in Interaction Modells die drei nachfolgend beschriebenen Schritte einhalten.

\subsection{Schritt 1: Status Quo}

Für die praktische Anwendung muss zuerst der aktuelle Status Quo aufgenommen werden, d.h. wie verhält sich das Unternehmen - insbesondere die eingesetzte IT auf den verschiedenen Ebenen gegenüber den Kunden. Es gilt sich zu vergegenwärtigen, welche Interaktionen getätigt werden, wie die IT die Interaktionen konkret unterstützt, wie diese ausgestaltet sind und welche Ziele dabei verfolgt werden. Beispielhafte Fragestellungen sind: 
- Welche Interaktionen gibt es aktuell zwischen dem Unternehmen und dem Kunden?

- Wie wurde IT in diese Interaktionen integriert? Welchen nicht-monetären Wert liefert sie dabei für den Kunden und das Unternehmen?

- Gibt es Limitierungen, die auf den Einsatz einer Technologie zurückzuführen sind? Kann ich diese Limitierungen durch den Einsatz anderer Technologien aufheben?

In diesem Zuge sollten auch Faktoren wie Churn, Abbrüche in Customer Journeys und fehlende Wiederkäufe analysiert werden. Alleine die Fokussierung auf die Interaktion an sich führt zum Hinterfragen von eingefahrenen Verhaltensweisen.

\subsection{Schritt 2: Handlungsfelder identifizieren}

Nun gilt es die einzelne Interaktion auf Basis des vorgestellten Modells zu überprüfen. Können digitale Interaktionsservices auf den drei Ebenen einen Wert erzeugen oder werden Ebenen bisher gar nicht oder nur rudimentär berücksichtigt. Dabei muss auch eruiert werden, was potenziell eine positive Wertegenerierung in der jeweiligen Phase verhindert. Im Ergebnis werden Interaktionen bewertet, um Handlungsfelder zu identifizieren.

\subsection{Schritt 3: Interaktionen aktiv gestalten}

Schlussendlich gilt es,

a) die aktuellen Interaktionen gemäß den identifizierten Herausforderungen anzupassen und

b) neue Interaktionen mit Wert zu gestalten.

In der Tab. 1 finden sich exemplarisch einige Fragestellungen, die es sich im Rahmen der Gestaltung der Interaktionen zu stellen gilt. Diese sind nicht als abschließend zu betrachten und individuell auf verschiedene Situationen anzupassen und zu erweitern.

Bei den Kompetenzen, die ein Anbieter einem Kunden im Rahmen der Schaffung des Value in Interaction anbietet, handelt es sich nicht um die, die er im Rahmen seiner Value Proposition anbietet. Stattdessen muss er weitere Kompetenzen ausprägen, um qualitativ hochwertige, IT-gestützte Interaktionen zu gestalten. Dazu gehören die in der Tab. 1 genannten Kompetenzen, die sich konkret beispielsweise auf die Anwendung von Wissen beziehen oder die IT-gestützte Integration der Fähigkeiten in der Kollaboration, dem Problemverständnis, der Lösungskompetenz oder Authentizität beinhalten und auch Fähigkeiten der guten Beratung, des Kuratierens oder der Vermittlung durch IT-Systeme einschließen. Dazu gehört es beispielsweise auch IT-gestützt emotionale Bedürfnisse anderer Akteure zu erkennen, ihr Vertrauen zu erlangen und, z.B. auf Basis von Wissen, darauf reagieren zu können. So kann eine gezielte Interaktion die Reaktion auf eine erfasste Emotion sein und auf der Matching-Ebene dazu beitragen, dass die für die jeweilige Situation bestmöglichen Servicebestandteile gewählt werden. Dies hat einen Einfluss auf die Beziehungsebe- 
Tab. 1 Beispielhafte Fragestellungen für die praktische Umsetzung

\begin{tabular}{|c|c|c|c|}
\hline Ebene & Zielstellung & Kompetenzen & Beispielhafte Fragestellungen \\
\hline Beziehungsebene & $\begin{array}{l}\text { Aufbau eines } \\
\text { positiven Bezie- } \\
\text { hungswert und } \\
\text { Überführung in } \\
\text { langfristige Be- } \\
\text { ziehung }\end{array}$ & $\begin{array}{l}\text { Kollaborations- } \\
\text { kompetenzen, } \\
\text { soziale Kompe- } \\
\text { tenzen }\end{array}$ & $\begin{array}{l}\text { Wie spreche ich den Kunden an } \\
\text { (z. B. Du, Sie)? } \\
\text { Welche Rolle möchte ich für den } \\
\text { Kunden erfüllen (z. B. Vermittler } \\
\text { oder Problemlöser)? } \\
\text { Welche Elemente kann ich bei der } \\
\text { IT-gestützten Interaktion nutzen, um } \\
\text { Vertrauen aufzubauen? } \\
\text { Wie muss ich das UI gestalten (z. B. } \\
\text { Bildwelten), damit sich der Kunde } \\
\text { wohl fühlt? } \\
\text { Welche IT-Systeme sind passend } \\
\text { (z. B. Chatbot, Instagram)? } \\
\text { Wie kann bspw. der Chatbot An- } \\
\text { teilnahme oder Einfühlsamkeit } \\
\text { signalisieren? } \\
\text {... }\end{array}$ \\
\hline Matching-Ebene & $\begin{array}{l}\text { Auswahl der } \\
\text { richtigen Service- } \\
\text { bestandteile mit } \\
\text { größtmöglicher } \\
\text { Dichte, um Mat- } \\
\text { ching-Wert zu } \\
\text { erhöhen }\end{array}$ & $\begin{array}{l}\text { Matching Kompe- } \\
\text { tenzen }\end{array}$ & $\begin{array}{l}\text { Was ist das Kundenproblem? Was } \\
\text { erwartet der Kunde von mir? Wie } \\
\text { kann ich das IT-gestützt herausfin- } \\
\text { den (z. B. Kundendaten, Beobach- } \\
\text { tung/Tracking)? } \\
\text { Welche Methoden und Kanäle nutze } \\
\text { ich, um das bestmögliche Service- } \\
\text { angebot (z. B. Inspiration, Bera- } \\
\text { tungsgespräch) für den Kunden } \\
\text { zusammenstellen zu können? } \\
\text { Wie kann ich die emotionale Lage } \\
\text { des Kunden einschätzen (z. B. IT- } \\
\text { gestützte Emotionserkennung und } \\
\text { Reaktion) und die passenden Inter- } \\
\text { aktionen gestalten (z. B. Emotions- } \\
\text { Reaktions-Guidelines)? } \\
\text {... }\end{array}$ \\
\hline Dienstleistungsebene & $\begin{array}{l}\text { Bestmögliche } \\
\text { Durchführung der } \\
\text { Dienstleistung, } \\
\text { um Dienstleis- } \\
\text { tungswert zu } \\
\text { maximieren }\end{array}$ & $\begin{array}{l}\text { Service-Kompe- } \\
\text { tenzen }\end{array}$ & $\begin{array}{l}\text { Habe ich alle Kompetenzen, die } \\
\text { es braucht, um das bestmögliche } \\
\text { Serviceangebot umzusetzen? } \\
\text { Wie kann ich die Dienstleistung } \\
\text { wertvoller gestalten und welchen } \\
\text { Anteil kann dabei der Einsatz von IT } \\
\text { leisten? } \\
\text { Habe ich Kompetenzen, die für } \\
\text { den Kunden im Prozess besonders } \\
\text { wertvoll erscheinen sodass ich dem- } \\
\text { entsprechend mein Serviceangebot } \\
\text { auf der Matching-Ebene nochmals } \\
\text { anpassen kann? }\end{array}$ \\
\hline
\end{tabular}


ne, da die emotionale Situation das Verhalten und die Zufriedenheit der Akteure stark beeinflusst (van Dolen et al. 2004). IT-Systeme, die es den Unternehmen beispielsweise erlauben die aktuelle Gemütslage des anderen Akteurs zu eruieren (Stichwort: Emotionserkennung und passende Reaktion) können hier Wertschöpfungsprozesse unterstützen.

\section{Fazit und Ausblick}

Im Rahmen dieses Beitrags wurde herausgearbeitet, dass Interaktionen der Klebstoff zwischen Akteuren und Voraussetzung für das Schaffen von Wert für die teilnehmenden Akteure sind. Eine steigende Anzahl an Interaktionen auf Basis neuer Interaktionsmöglichkeiten - geschaffen durch die IT als Mediator - unterstreichen diese Wichtigkeit. Es wird vorgeschlagen, die Interaktion in den Mittelpunkt der Gestaltung von IT-gestützten Services zu stellen und einen Value in Interaction einzuführen. Dieser Wert setzt sich aus dem Beziehungswert, dem Matching-Wert und dem Dienstleistungswert zusammen. Dementsprechend reicht es als Anbieter nicht mehr aus, ausschließlich über Kompetenzen für die Bereitstellung seines Dienstleistungsangebots $\mathrm{zu}$ verfügen. Interaktionen müssen aktiv gestaltet werden, um einen (positiven) Wert für alle teilnehmenden Akteure zu generieren. So bietet sich die Chance, dass neue Werte entstehen oder bestehende beeinflusst werden, neue Interaktionsräume entdeckt und genutzt werden und die Beziehung zwischen den Akteuren angereichert wird.

Im weiteren Verlauf ist der empirische Nachweis der theoretisch hergeleiteten Wirkungsweise zu erbringen. Zudem sollen auf Basis der in Abschn. 4 dargestellten Schritte konkrete Guidelines aufgebaut und Design Patterns für das aktive Gestalten der Interaktionen (Schritt 3) entwickelt werden. Da die Anforderungen grundsätzlich sehr individuell sein werden, sollen die aktuell in der Wirtschaftsinformatik diskutierten Technologien und deren Anwendungen wie Emotionserkennung, Personality Mining, KI oder Chatbots in Bezug auf das Value in Interaction Modell überprüft werden. Es soll eruiert werden, wie diese Technologien eingebunden werden müssen, um Wert zu erzeugen und welche Voraussetzungen dafür erfüllt sein müssen.

Funding Open Access funding provided by Projekt DEAL.

Open Access Dieser Artikel wird unter der Creative Commons Namensnennung 4.0 International Lizenz veröffentlicht, welche die Nutzung, Vervielfältigung, Bearbeitung, Verbreitung und Wiedergabe in jeglichem Medium und Format erlaubt, sofern Sie den/die ursprünglichen Autor(en) und die Quelle ordnungsgemäß nennen, einen Link zur Creative Commons Lizenz beifügen und angeben, ob Änderungen vorgenommen wurden.

Die in diesem Artikel enthaltenen Bilder und sonstiges Drittmaterial unterliegen ebenfalls der genannten Creative Commons Lizenz, sofern sich aus der Abbildungslegende nichts anderes ergibt. Sofern das betreffende Material nicht unter der genannten Creative Commons Lizenz steht und die betreffende Handlung nicht nach gesetzlichen Vorschriften erlaubt ist, ist für die oben aufgeführten Weiterverwendungen des Materials die Einwilligung des jeweiligen Rechteinhabers einzuholen.

Weitere Details zur Lizenz entnehmen Sie bitte der Lizenzinformation auf http://creativecommons.org/ licenses/by/4.0/deed.de. 


\section{Literatur}

van Dolen W, de Ruyter K, Lemmink J (2004) An empirical assessment of the influence of customer emotions and contact employee performance on encounter and relationship satisfaction. J Bus Res 57:437-444. https://doi.org/10.1016/S0148-2963(02)00277-1

Fyrberg A, Jüriado R (2009) What about interaction?: Networks and brands as integrators within servicedominant logic. J Serv Manag 20:420-432. https://doi.org/10.1108/09564230910978511

Grönroos C (2011) Value co-creation in service logic: a critical analysis. Mark Theory 11:279-301. https:// doi.org/10.1177/1470593111408177

Grönroos C, Ojasalo K (2004) Service Productivity: Toward a Conceptualisation of the Transformation of inputs. Swed Sch Econ Bus Adm-Work Pap 25

Grönroos C, Ravald A (2009) Marketing and the logic of service: value facilitation, value creation and cocreation, and their marketing implications. Hanken Sch Econ Work Pap, S 1-38

Grönroos C, Voima P (2013) Critical service logic: making sense of value creation and co-creation. J Acad Mark Sci 41:133-150. https://doi.org/10.1007/s11747-012-0308-3

Heinemann G (2013) Digitale Revolution im Handel - steigende Handelsdynamik und disruptive Veränderung der Handelsstrukturen. In: Heinemann G, Haug K, Gehrckens M (Hrsg) Digitalisierung des Handels mit ePace: Innovative E-Commerce-Geschäftsmodelle und digitale Zeitvorteile. Springer, Wiesbaden, S 3-26

Iyengar SS, Lepper MR (2000) When choice is demotivating: can one desire too much of a good thing? J Pers Soc Psychol 79:995-1006. https://doi.org/10.1037//0022-3514.79.6.995

Lusch RF, Nambisan S (2015) Service innovation: a service-dominant logic perspective. Mis Q 39:155-175. https://doi.org/10.25300/MISQ/2015/39.1.07

Normann R (2001) Reframing business: when the map changes the landscape. Wiley, Chichester

Ravald A, Grönroos C (1996) The value concept and relationship marketing. Eur J Mark 30:19-30. https:// doi.org/10.1108/03090569610106626

Schwartz B (2004) The paradox of choice. HarperCollins Publishers, New York

Vargo SL, Lusch RF (2008) Service-dominant logic: continuing the evolution. J Acad Mark Sci 36:1-10. https://doi.org/10.1007/s11747-007-0069-6

Wan Y, Menon S, Ramaprasad A (2003) How it happens: a conceptual explanation of choice overload in Online decision-making by individuals. Decis Support Syst. https://doi.org/10.4067/S071818762009000300008 